Original Article (short paper)

\title{
Dose-response of neuromuscular and anaerobic performance to whole-body vibration
}

\author{
Núbia Carelli Pereira de Avelar ${ }^{1}$ (D), Daniele Maria Silva Viana ${ }^{2}$ (D) , Vanessa Gonçalves César Ribeiro ${ }^{2}$ (D) , \\ Fabiano Salvador ${ }^{2}$ (D) , Ana Cristina Rodrigues Lacerda ${ }^{2}$ \\ ${ }^{1}$ Universidade Federal de Santa Catarina, Departamento de Ciências da Saúde, Laboratório de \\ Reumatologia, Envelhecimento e Recursos, Araranguá, SC, Brasil; ${ }^{2}$ Universidade Federal dos Vales do \\ Jequitinhonha e Mucuri, Faculdade de Ciências Biológicas, Laboratório de Fisiologia do Exercício, Diamantina, MG, Brasil
}

\begin{abstract}
Aims: The present study was designed to investigate the dose-response relationship of muscular performance and anaerobic power to whole-body vibration (WBV). Methods: Nine recreationally males were subjected to five experimental conditions: A) Squat exercise (SE) without WBV; B) WBV1 [31.55 m. $\mathrm{s}^{-2}$; C) WBV2 [159.73 m. $\left.\mathrm{s}^{-2}\right]$; (D) WBV3 [319.45 m.s. $\mathrm{s}^{-2}$; andE) WBV4 [567.91 $\left.\mathrm{m} . \mathrm{s}^{-2}\right]$. Before and after the experimental procedures, muscleperformance was assessed by a vertical jump test and handgrip strength test and anaerobic power by Wingate anaerobic test (WT). Results: WBV2 and WBV3 increased jump relative power $[\Delta=0.92 \pm 0.89 \mathrm{~W} / \mathrm{Kg}(+1.97 \%)$ and $\Delta=1.29 \pm 1.79 \mathrm{~W} / \mathrm{Kg}(+2.77 \%)$, respectively; $P=0.02$; effect size $=0.92 ;$ power $=0.87]$ and height $[\Delta=0.96 \pm 0.73 \mathrm{~cm}(2.80 \%)$ and $\Delta=1.61 \pm 2.36 \mathrm{~cm}(4,57 \%)$, respectively; $P=<0.01$; effect size $=0.96$; power $=0.99]$ compared to the $\operatorname{SE}[\Delta=-1.28 \pm 0.81 \mathrm{~cm}(-3.55 \%)]$. There was no dose-response relationship of handgrip strength to WBV. WBV2 improved WT relative power (power/body weight) compared to the other experimental conditions $[\Delta=0.61 \pm 0.36 \mathrm{~W} / \mathrm{Kg}(+5.25 \%) ; P<0.01$; ; effect size $=0.98$; power $=$ $0.99]$, and WT relative work was higher in WBV3 $[\Delta=1.86 \pm 5.46 \mathrm{KJ} / \mathrm{Kg}(+0.70 \%)$ condition compared to WBV1[ $\Delta=$ $-6.71 \pm 4.03 \mathrm{KJ} / \mathrm{Kg}(-2.49 \%)(P=0.03)$. Conclusion: These results suggest that lower limb muscular performance and anaerobic power are responsive to vibration accelerations WBV2 (159.73 m.s-2) and WBV3 (319.45 m.s-2).
\end{abstract}

Keywords: physical functional performance; athletes; warm-up exercise.

\section{Introduction}

In recent studies, whole-body vibration (WBV) has been employed as an alternative method of exercise to improve the physical performance of athletes ${ }^{1-3}$. Several studies have demonstrated the positive impact of $\mathrm{WBV}$ on tests of muscle power ${ }^{4,5}$, muscle strength $^{5-7}$, and flexibility ${ }^{8}$. However, literature is not unanimous, and there are studies that demonstrate that the addition of an acute vibratory stimulus to exercise is not able to produce additional improvements in physical and functional performance ${ }^{9-11}$.

During WBV, the gravitational load (hypergravity condition) imposed on the neuromuscular system is determined by the peak-to-peak displacement - the displacement from the lowest to the highest point - and the frequency of the vibrating plate. Therefore, the accelerations that have been reported in the literature have been realized by manipulating various combinations of frequency (from 20 to $60 \mathrm{~Hz}$ ) and peak-to-peak displacement (from 2 to $10 \mathrm{~mm})^{12-14}$. Although there have been attempts to examine the acute dose-response effect of vibration frequency on muscle performance in previous investigations ${ }^{15-18}$, the results are controversial, and to the best of our knowledge, no study has evaluated the dose-response relationship of vibration acceleration on muscular performance.

It is well known in the scientific literature that the vibratory stimulus is damped by lower limb muscles. However, it is unknown whether the magnitude of this stimulus reaches the upper limbs and what it is the influence of the acceleration on this muscle group performance. Marín et al. ${ }^{19}$, demonstrated that WBV applied underfoot increases muscle performance of elbow extensors, suggesting that the muscle response was associated with the vibratory stimulus. However, the authors did not verify if there is a dose-response relationship of the vibratory stimulus on the muscle performance of the upper limb.

The use of WBV as an alternative method of exercise to improve the physical performance of athletes should be considered for coaches and practitioners of sports activities on high-intensity performance since it is able to promote an increase in muscle power ${ }^{1}$. Furthermore, WBV seems to promote a more effective heating muscle than traditional forms of active and passive warm-up ${ }^{20}$. Therefore, squatting with acute WBV may be an alternative and faster method to increase muscle temperature and enhance the performance of sporting activities. However, the exact protocol to be used for its practical application is still unclear, as is the precise nature of the possible usefulness of vibration in field-based settings.

Since lower and upper limbs performance could be associated with the acceleration produced by the vibratory platform, the present study was designed to investigate the dose-response relationship of muscular performance and anaerobic power to WBV. Thus, in the present investigation, we tested the hypothesis that in regularly and recreationally trained healthy men, muscular performance is dose-related to WBV acceleration in a sine wave fashion. Moreover, this dose-response effect on muscle performance is not evident in the upper limbs. 


\section{Methods}

\section{Subjects}

Nine regularly and recreationally trained healthy men (age: $23.00 \pm 5.55$ years old, height: $1.72 \pm 0.05 \mathrm{~cm}$, body mass: 66.57 $\pm 8.82 \mathrm{~kg}$, maximal oxygen consumption: $55.53 \mathrm{mLO}_{2}{ }^{*} \mathrm{~kg}^{*} \mathrm{~min}^{-1}$ ), volunteered to take part in this study. All participants were engaged in national competitions (running and cycling), and their training frequently targeted neuromuscular components (power, strength, and muscle endurance). None of them had reported muscle injuries in the last 2 months. Participants were asked to report the use of drugs during the experimental protocol; moreover, were instructed to refrain from physical activity, consumption of alcohol, and caffeine for 24 hours before testing. Volunteers were asked to maintain the same dietary habits, eight hours of sleep, and consumption of $500 \mathrm{ml}$ of water two hours before each experimental condition. On the test day, subjects were asked about compliance with the recommendations above and for possible complications or changes in a daily routine that occurred.

The participants were notified about the potential risks involved in the study and gave their written informed consent. This study was approved by the Federal University of Jequitinhonha and Mucuri Valleys (CCAE: 33275320.8.0000.5108).

\section{Study design and exercise protocol}

In this cross-over study, each one of the 9 participants performed all 5 experimental conditions. The order of allocation of the experimental conditions was carried out using, for each volunteer, the EXCEL program with the "random function between" to define the sequence of the different experimental conditions (1 to 5).

The study consisted of a preliminary session and the experimental procedures (Figure 1). All procedures were performed under a thermoneutral condition: $21-24^{\circ} \mathrm{C}$ dry temperature and 50 to $75 \%$ relative humidity, as recommended by Clark and Edholm ${ }^{21}$.

\section{Preliminary session}

A preliminary session was performed 7 days before the experimental procedures and consisted of anthropometric measurements (weight and height) followed by maximal oxygen consumption determination, as well as familiarization with the experimental procedures.

\section{Procedures}

\section{Pretests}

To assess baseline data (pretest), the volunteers remained at rest for 5 minutes before the Wingate anaerobic test (WT) (pretest) followed by 20 minutes at rest for recovery ${ }^{22}$. Thereafter, the volunteers performed the jump test, followed by the handgrip strength test, and immediately after these tests, they were submitted to 1 of the 5 experimental conditions.

\section{Experimental conditions}

The experimental conditions consisted of the following:

1) Squat exercise (SE) without WBV $\left[0 \mathrm{~m}^{-2} \mathrm{~s}^{-2}\right.$;

2) WBV1 $\left(20 \mathrm{~Hz} / 2 \mathrm{~mm}: 31.55 \mathrm{~m} . \mathrm{s}^{-2}\right)$;

3) WBV2 (45Hz/2mm: $\left.159.73 \mathrm{~m} \cdot \mathrm{s}^{-2}\right)$;

4) WBV3 (45Hz/4mm: $\left.319.45 \mathrm{~m} \cdot \mathrm{s}^{-2}\right)$; and

5) WBV4 (60Hz/4mm: $\left.567.91 \mathrm{~m} . \mathrm{s}^{-2}\right)$.

These experimental conditions were imposed at intervals of 24 hours in a randomized order. To minimize the circadian influence, the participants performed all interventions at the same time each day ${ }^{20}$.

The doses of acceleration imposed by the WBV device were determined by the peak-to-peak displacement and frequency of the oscillations. The magnitude is reported in meters per second squared $\left(\mathrm{m} . \mathrm{s}^{-2}\right)$ and represents the stimulus intensity during exercise; this value is reported as the number of times the intensity exceeds gravitational acceleration $(\mathrm{g})$ or the value of maximum acceleration $\left(\mathrm{A}_{\max }\right)^{23}$. By definition, $A_{\max }$ refers to the peak rate of change of velocity of the vibration plate. The relationship of $A_{\max }$ with vibration frequency and peak-to-peak displacement was calculated using the following equation: $A_{\max }=a_{\text {peak-to-peak }}(2 д f)^{2}$, where "a $a_{\text {peak-to-peak }}$ " and "f" represent peak-to-peak displacement and frequency, respectively ${ }^{24}$.

The vibratory stimulus was performed using a commercially produced vibration platform (FitVibe, GymnaUniphy NV, Bilzen, Belgium) that produces vertical sinusoidal vibrations in both legs while the platform moves predominantly in the vertical direction $^{13}$. There was no vibratory stimulus in the condition of SE without WBV; the individuals remained on the vibration platform without the operation of the apparatus.

For the SE, the volunteers were asked to stand barefoot on the vibration platform ${ }^{19}$ with their feet apart at a distance of $28 \mathrm{~cm}$ and had to perform SE (semi-flexion of the knee from $10^{\circ}$ to $90^{\circ}$ ). The flexing to $90^{\circ}$ was measured for each volunteer using a universal goniometer before starting the exercise series, and a barrier was placed in the gluteal region to limit the degree of knee flexion; therefore, all the volunteers flexed their knees to an angle of $90^{\circ 25}$.

To control the time of each squat, an examiner instructed the individuals to bend their knees to an angle of 90 for 3 seconds and then flex them to an angle of 10 for 3 seconds over a period of 5 minutes. In addition, the volunteers spent 1 second at the end of the concentric and eccentric phase, therefore the repetition lasted 8 seconds. Therefore, 37 repetitions were performed in 5 minutes. The participants were also instructed on body mechanics (i.e., the correct position of their feet on the platform and the positions of their spine, arms, and head) ${ }^{1}$. The timing of the SE was set using a digital metronome. 


\section{Posttests}

Three minutes after the experimental procedure, the volunteers performed the jump test, followed by the handgrip strength test. Thereafter, the volunteers remained at rest for 7 minutes and repeated the same experimental condition followed by the WT 3 minutes after.

All tests were conducted by the same examiner, and the intervals between the tests and the experimental conditions were based on the study of Avelar et al. ${ }^{1}$ that used 3 minutes after performing SE with WBV to perform sprint cycling performance. Sale $^{26}$ suggested that at least 3 minutes of rest are required to eliminate fatigue before a test.

The interval of approximately 7 minutes was based on a pilot study that demonstrated that this period was sufficient for the body return to homeostasis after a vibratory stimulus. Furthermore, Cochrane et al. ${ }^{3}$ showed that residual effects from vibration occurred no later than 5 minutes after the cessation of a vibratory stimulus.

\section{Wingate anaerobic test}

The Wingate anaerobic test (WT) was chosen because it measures power, relative work (work/body weight), and pedaling cadence of lower limbs, which are variables that compose the muscular performance ${ }^{22}$. Briefly, during the test, the volunteer was requested to cycle against a predetermined resistance (7.5\% of body weight) as rapidly as possible for 30 seconds. Power output was recorded online by a computer connected to a Monark standard cycle ergometer (Maxx, Hidrofit, Belo Horizonte, Brazil) during the 30-s sprint test. To record power output, MCE model software (Multi-Cycle Ergometer, 2.3 version, Warsaw Sports Institute, Poland) was used.

\section{Jump Test}

During the jump test, jumping mat pressure (Jump System 1.0, Cefise, Brazil), was used to measure the relative power [absolute power/body weight $(\mathrm{W} / \mathrm{Kg})$ ] and height of the jump. Jumping mat pressure corresponds to a pair of pressure sensors coupled to the plantar surface of the forefoot and barefoot of the individuals.

The subjects were instructed to jump as high as possible in three vertical jumps with a 10-second interval between jumps. The jumps began from a squat position with the knees at $100^{\circ}$ of flexion ${ }^{27}$, the hands resting on the iliac crest (in an attempt to remove impulsion resulting from arm oscillations) and the feet spaced $18 \mathrm{~cm}$ apart. Afterward, the height of the jump and the power output were calculated using the formulas shown below ${ }^{28}$ :

Height of the Jump: $1 / 8 \mathrm{gt}^{2}$

Where $g$ is the acceleration of gravity $\left(9.81 \mathrm{~ms}^{-2}\right)$, and $t$ is the jump air time.

Power Output: $60.7 *$ (height of the jump $+45.3 *$ (body mass) -2055
The highest values of relative power and height of jump were considered for analysis.

\section{Handgrip strength test}

The handgrip strength test was performed by the dominant upper limb using a hand dynamometer (Crown, Filizola, Brazil). The instrument provides one quick and straightforward reading of isometric force and is adaptable to different hand $\operatorname{sizes}^{29}$. The test was repeated three times with intervals of 60 seconds between each run, and the highest value was reported. The volunteers were instructed to perform the test during expiration without performing the Valsalva maneuver, and they received standardized verbal encouragement during the test.

The subject remained seated in a chair without armrests, keeping a straight trunk, feet flat on the floor and knees at $90^{\circ}$ flexion. Additionally, the subject maintained an adducted shoulder, elbow at $90^{\circ}$ flexion, forearm pronated, and wrist in half neutral to a slight extension. The arm was suspended and placed on the hand dynamometer. This position was sustained by the examiner.

\section{Intraclass Correlation Coefficient of Measurements}

Prior to the beginning of the study, the intra-examiner reliabilities for the WT, Jump Test, and Handgrip strength tests were calculated. For this, ten volunteers were submitted to a WT, jump test, and handgrip strength test on two separate days with an interval of at least 24 hours between the tests. Intra-class correlation coefficients (ICC) were 0.994 (95\% CI 0.978 - 0.998), 0.967 (95\% CI $0.927-0.985)$, and 0.968 (95\% CI $0.929-0.985)$, respectively.

\section{Statistical Analysis}

The SPSS ${ }^{\circledR}$ statistical software program (IBM ${ }^{\circledR}$, Chicago, IL, USA), version 19.0, was used for the statistical analysis. The data were expressed as the means and standard deviation. The significance level was defined as $P \leq 0.05$. Statistical analysis was performed between the groups through variation $(\Delta)$ within each group, where Post Value - Pre-Value $=$ Delta Value $(\Delta)$. The Shapiro-Wilk test was used to evaluate the normalcy of the data [Delta Value $(\Delta)$ ]. Because the data were normally distributed for the dependent variables, parametric tests were used for statistical analysis. A repeated measure one-way ANOVA was performed to test the difference between conditions, if differences were found a Tukey's post hoc was used. To check the size of the differences between the pre- and post-experimental periods, we analyzed the magnitude of the effects. Thus, with a magnitude of effect analysis, it is possible to identify whether observed differences are small, moderate, or large $\mathrm{e}^{30}$. 


\section{Experimental Procedures}

Preliminary Session
7 Days

\section{Experimental conditions:}
A) Squat exercise (SE) without WBV $\left(0 \mathrm{~m} \cdot \mathrm{s}^{-2}\right)$;
B) WBV1 $\left(20 \mathrm{~Hz} / 2 \mathrm{~mm}: 31.55 \mathrm{~m} . \mathrm{s}^{-2}\right)$;
C) WBV2 $\left(45 \mathrm{~Hz} / 2 \mathrm{~mm}: 159.73 \mathrm{~m} \cdot \mathrm{s}^{-2}\right)$;
D) WBV3 $\left(45 \mathrm{~Hz} / 4 \mathrm{~mm}: 319.45 \mathrm{~m} . \mathrm{s}^{-2}\right)$;
E) WBV4 $\left(60 \mathrm{~Hz} / 4 \mathrm{~mm}: 567.91 \mathrm{~m} \cdot \mathrm{s}^{-2}\right)$.

Figure 1 - Study flow chart.

\section{Results}

All of the participants completed all of the experimental procedures of all of the interventions. The data obtained in tests of muscular performance before the experimental conditions showed no difference from the baseline data (Table 1).

WBV2 and WBV3 (159.73 m. $\mathrm{s}^{-2}$ and $\left.319.45 \mathrm{~m} . \mathrm{s}^{-2}\right)$ increased jump relative power $[(\Delta=0.92 \pm 0.89 \mathrm{~W} / \mathrm{Kg}(+1.97 \%)$ and $\Delta=1.29 \pm 1.79 \mathrm{~W} / \mathrm{Kg}(+2.77 \%) ; \mathrm{P}=0.0204$; effect size: 0.92 ; power: 0.87$])$ and height $[(\Delta=0.96 \pm 0.73 \mathrm{~cm}(2.80 \%)$ and $\Delta=1.61 \pm 2.36 \mathrm{~cm}(4,57 \%) ; \mathrm{P}<0.01$; effect size: 0.96 ; power: $0.99])$ compared to the SE $[\Delta=-1.28 \pm 0.81 \mathrm{~cm}(-3.55 \%)]$ without WBV condition. Moreover, jump relative power was higher after a vibration stimulus of $319.45 \mathrm{~m} \cdot \mathrm{s}^{-2}$ (WBV3) compared to a vibration stimulus of $31.55 \mathrm{~m} . \mathrm{s}^{-2}$ (WBV1) ( $P=0.004$ ) (Figure 2). There was no significant effect on handgrip strength of the vibratory stimulus during SE $(P>$ 0.05) (Figure 3).

WBV2 improved WT relative power compared to the other experimental conditions $[\Delta=0.61 \pm 0.36 \mathrm{~W} / \mathrm{Kg}(+5.25 \%) ; P$ $<0.01$; ; effect size: 0.98; power: 0.99], and WT relative work was higher in WBV3 $[\Delta=1.86 \pm 5.46 \mathrm{KJ} / \mathrm{Kg}(+0.70 \%)$ condition compared to $\mathrm{WBV} 1[\Delta=-6.71 \pm 4.03 \mathrm{KJ} / \mathrm{Kg}(-2.49 \%)$ ( $P=0.03)$ (Table 2). 
A

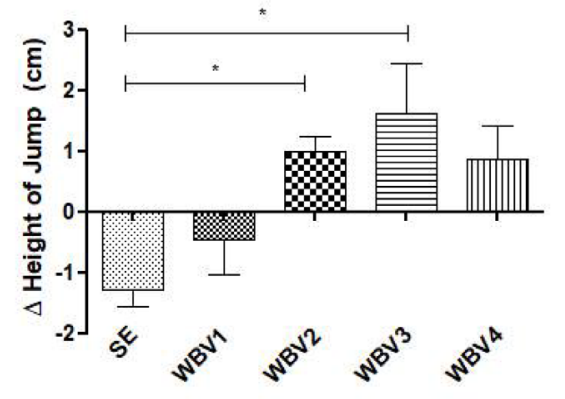

B

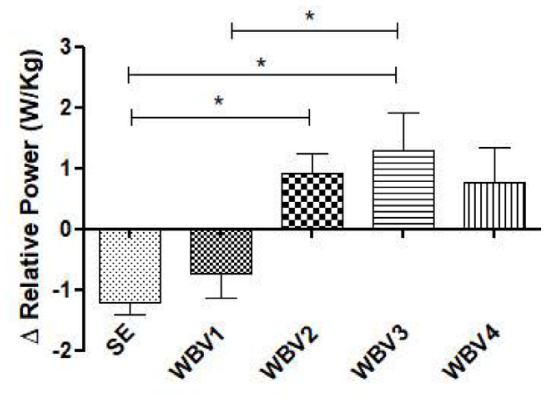

Figure 2 - The change ( $\Delta=$ Post - Pretest) in jump height (A) and jump relative power (B) among the experimental conditions. * Significantly different with $\mathrm{P}<$ $0.05(\mathrm{~N}=9)$.

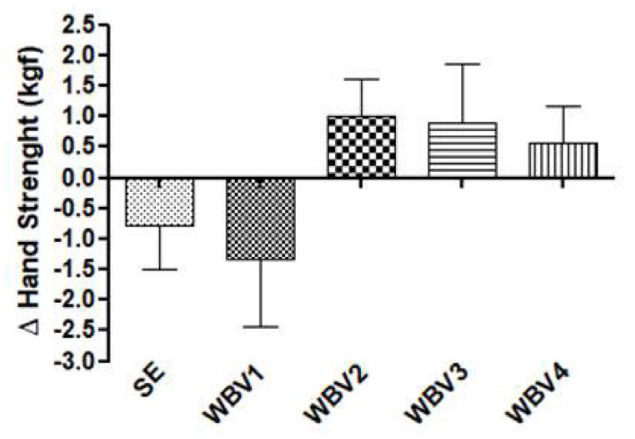

Figure 3 - The change ( $\Delta=$ Post - Pretest) in handgrip strength among the experimental conditions $(N=9)$. 
Table 1 - Baseline data (pretest) of muscular performance for the experimental conditions (N=9).

\begin{tabular}{|c|c|c|c|c|c|c|}
\hline & $\begin{array}{c}\text { SE } \\
0 \mathrm{~m} . \mathrm{s}-2 \\
\end{array}$ & $\begin{array}{c}\text { WBV1 } \\
31.55 \text { m.s-2 }\end{array}$ & $\begin{array}{c}\text { WBV2 } \\
159.73 \text { m.s-2 }\end{array}$ & $\begin{array}{c}\text { WBV3 } \\
319.45 \text { m.s-2 }\end{array}$ & $\begin{array}{c}\text { WBV4 } \\
567.91 \text { m.s-2 }\end{array}$ & $P$ \\
\hline \multicolumn{7}{|l|}{ Jump Test } \\
\hline Jump Height (cm) & $36.04 \pm 3.80$ & $36.19 \pm 2.63$ & $35.24 \pm 4.69$ & $35.24 \pm 3.51$ & $35.88 \pm 4.56$ & 0.98 \\
\hline Relative Power (W/Kg) & $47.19 \pm 3.56$ & $47.76 \pm 2.70$ & $47.46 \pm 4.77$ & $46.63 \pm 3.37$ & $47.06 \pm 4.09$ & 0.91 \\
\hline \multicolumn{7}{|l|}{ Handgrip Strength Test } \\
\hline Strength (Kgf) & $36.5 \pm 6.02$ & $35.25 \pm 7.87$ & $35.5 \pm 7.07$ & $36.25 \pm 5.85$ & $35.25 \pm 5.47$ & 0.25 \\
\hline \multicolumn{7}{|l|}{ Wingate Anaerobic Test } \\
\hline Mean Power (W) & $\begin{array}{l}600.00 \pm \\
62.26\end{array}$ & $\begin{array}{l}584.86 \pm \\
77.46\end{array}$ & $\begin{array}{c}593.00 \pm \\
69.99\end{array}$ & $\begin{array}{l}565.71 \pm \\
27.52\end{array}$ & $\begin{array}{c}570.29 \pm \\
27.01\end{array}$ & 0.59 \\
\hline Peak Power (W) & $\begin{array}{c}811.57 \pm \\
101.82\end{array}$ & $\begin{array}{c}787.14 \pm \\
108.88\end{array}$ & $\begin{array}{c}746.71 \pm \\
87.12\end{array}$ & $\begin{array}{c}757.00 \pm \\
81.28\end{array}$ & $812.86 \pm 108.07$ & 0.71 \\
\hline Relative power (W) & $12.00 \pm 0.47$ & $11.82 \pm 0.53$ & $11.61 \pm 1.03$ & $12.07 \pm 0.88$ & $12.21 \pm 0.97$ & 0.99 \\
\hline Total work (KJ) & $17.60 \pm 1.82$ & $17.92 \pm 2.12$ & $17.11 \pm 0.77$ & $16.97 \pm 0.77$ & $17.87 \pm 2.10$ & 0.62 \\
\hline Relative work (KJ) & $\begin{array}{c}272.71 \pm \\
15.66\end{array}$ & $\begin{array}{c}269.00 \pm \\
20.00\end{array}$ & $\begin{array}{c}266.43 \pm \\
13.16\end{array}$ & $\begin{array}{c}266.86 \pm \\
14.16\end{array}$ & $\begin{array}{c}267.86 \pm \\
14.36\end{array}$ & 0.96 \\
\hline Time to peak power (s) & $2.14 \pm 0.37$ & $2.19 \pm 0.25$ & $2.45 \pm 0.85$ & $2.27 \pm 0.55$ & $2.34 \pm 1.03$ & 0.89 \\
\hline
\end{tabular}

SE: squat exercise. WBV: whole-body vibration.

W/Kg: Watts/kilograms

Kgf: kilogram-force

W: Watts

KJ: Kilojoules

Table 2 - The change ( $\Delta=$ Post - Pretest $)$ in WT among the experimental conditions $(N=9)$.

\begin{tabular}{|c|c|c|c|c|c|c|}
\hline & $\begin{array}{c}\mathrm{SE} \\
0 \mathrm{~m} \cdot \mathrm{s}^{-2}\end{array}$ & $\begin{array}{c}\text { WBV1 } \\
31.55 \mathrm{~m}^{-2}\end{array}$ & $\begin{array}{c}\text { WBV2 } \\
159.73 \mathrm{~m} . \mathrm{s}^{-2}\end{array}$ & $\begin{array}{c}\text { WBV3 } \\
319.45 \mathrm{~m} . \mathrm{s}^{-2}\end{array}$ & $\begin{array}{c}\text { WBV4 } \\
567.91 \mathrm{~m} \cdot \mathrm{s}^{-2}\end{array}$ & $P$ \\
\hline Mean Power (W) & $\begin{array}{c}-10.14 \pm \\
9.62\end{array}$ & $\begin{array}{c}-13.74 \pm \\
9.95\end{array}$ & $\begin{array}{c}-2.43 \pm \\
13.78\end{array}$ & $\begin{array}{c}3.43 \pm \\
11.93\end{array}$ & $\begin{array}{c}-7.14 \pm \\
9.79\end{array}$ & 0.30 \\
\hline Peak Power (W) & $\begin{array}{l}-41.86 \\
\pm 22.88\end{array}$ & $\begin{array}{c}-38.00 \pm \\
26.80\end{array}$ & $\begin{array}{c}24.33 \pm \\
25.76\end{array}$ & $\begin{array}{l}3.78 \pm \\
28.83\end{array}$ & $\begin{array}{c}-8.57 \pm \\
15.35\end{array}$ & 0.11 \\
\hline Relative power (W) & $-0.63 \pm 0.37$ & $-0.56 \pm 0.39$ & $0.61 \pm 0.36 *$ & $-0.14 \pm 0.42$ & $-0.14 \pm 0.25$ & $<0.01$ \\
\hline Total work (KJ) & $-0.23 \pm 0.28$ & $-0.26 \pm 0.31$ & $-0.04 \pm 0.38$ & $0.08 \pm 0.30^{¥}$ & $-0.09 \pm 0.20$ & 0.04 \\
\hline Relative work (KJ) & $-4.71 \pm 4.75$ & $-6.71 \pm 4.03$ & $-1.43 \pm 5.97$ & $1.86 \pm 5.46^{¥}$ & $-1.86 \pm 3.02$ & 0.03 \\
\hline Time to peak power (s) & $0.04 \pm 0.23$ & $0.13 \pm 0.32$ & $-0.05 \pm 0.52$ & $-0.09 \pm 0.23$ & $-0.09 \pm 0.23$ & 0.71 \\
\hline
\end{tabular}

SE: squat exercise. WBV: whole body vibration.

* Significantly different from other intensities of acceleration, ¥ significantly different from SE + WBV $31.55 \mathrm{~m} . \mathrm{s}^{-2}$

\section{Discussion}

The main findings of the present study were that the acceleration doses of $159.73 \mathrm{~m} \cdot \mathrm{s}^{-2}$ and $319.45 \mathrm{~m} \cdot \mathrm{s}^{-2}$ (WBV 2 and WBV3) increased jump relative power and jump height, as well as augmenting relative power and relative work in the Wingate anaerobic test. These results point to the existence of a dose-response relationship in which muscle performance improves when exposed to a range of magnitudes of the vibratory stimulus.

Recent studies have produced conflicting results about the effect of acute WBV on muscle performance ${ }^{1,4,5,8,9-11,27}$. However, the wide range of maximum accelerations used in these studies 
$\left(52.7 \mathrm{~m} . \mathrm{s}^{-2}\right.$ to $\left.591.58 \mathrm{~m} . \mathrm{s}^{-2}\right)$ is worth mentioning. Some authors believe that the changes in neuromuscular performance ${ }^{12,31}$ resulting from vibratory stimuli are induced by momentary disturbances in the gravitational field that the body experiences during the WBV derived from the maximum acceleration. In other words, the protocols of WBV should be manipulated to obtain maximum acceleration values within the dose-response relationship to improve muscular performance.

It may be noted that the acceleration of $31.55 \mathrm{~m} \cdot \mathrm{s}^{-2}$ (WBV1) seems to be insufficient to generate a neuromuscular overload, and therefore, this frequency was not effective at improving performance. Accordingly, protocols that use low values of maximal acceleration may not be effective at promoting physiological effects capable of generating or maintaining the gain in physical performance, such as increased muscle temperature ${ }^{19,20}$, decreased joint and muscle resistance ${ }^{18}$, increased rate of nerve conduction $^{32}$, increased speed in metabolic processes ${ }^{33}$, or brain activation related to motor movement ${ }^{34}$. Furthermore, the placebo effect can be ruled out as providing a possible improvement in performance, as an acceleration of $31.55 \mathrm{~m} . \mathrm{s}^{-2}$ did not demonstrate an improvement in muscle performance despite volunteers reporting the feeling of transmission of WBV stimulus.

Values of maximal acceleration of approximately 159.73 $\mathrm{m} . \mathrm{s}^{-2}$ (WBV2) have been described in the current literature as being able to promote improvement in physical performance, especially in muscular power tests ${ }^{1,8,20}$. Cochrane et al. ${ }^{20}$ conducted a study in which changes in muscle temperature were obtained as a result of WBV in the same range and concluded that this intensity of vibration was effective at increasing power performance. However, it is noteworthy that other possible maximum acceleration values between 31.55 and $159.73 \mathrm{~m} . \mathrm{s}^{-2}$ can produce positive effects in terms of performance ${ }^{1,4-6,8,16,18,20,27}$. The literature shows that maximum accelerations close to 100.00 $\mathrm{m} . \mathrm{s}^{-2}$ may be sufficient to promote improvement in vertical jump tests $^{6,27}$. Accordingly, Cormie et al. ${ }^{27}$ verified the effectiveness of the WBV in the vertical jump using an acceleration value of $88.74 \mathrm{~m} . \mathrm{s}^{-2}$ and verified the effectiveness of the WBV in the vertical jump by approximately $0.7 \%$ after the vibratory stimulus $^{27}$. Furthermore, Siu et al. ${ }^{6}$ examined the immediate effects of two protocols with different vibration frequencies that yielded the same maximum acceleration $\left(106.75 \mathrm{~m} . \mathrm{s}^{-2}\right)$ on muscle peak torque and stiffness of knee extensor and flexor. Their experiment showed that WBV at frequencies of $26 \mathrm{~Hz}$ and $40 \mathrm{~Hz}$ precludes the decline in the concentric peak torque of the knee extensors observed after 10 bouts of 60 seconds of static half squats.

The vertical jump performance seems to respond positively to both accelerations of $159.73 \mathrm{~m} . \mathrm{s}^{-2}$ (WBV2) and 319.45 $\mathrm{m} . \mathrm{s}^{-2}$ (WBV3). In the present study, we observed increases in both height and relative power of jump after WBV using both doses of acceleration. These data reinforce the study of Avelar et al. ${ }^{1}$ in which the authors demonstrated that both intensities (159.73 m.s $\mathrm{s}^{-2}$ and $319.45 \mathrm{~m} . \mathrm{s}^{-2}$ ) of WBV were able to increase the height and relative power of jump similarly. In both studies, other factors that could influence muscle performance besides the magnitude of vibration acceleration were standardized, i.e., physical and anthropometric characteristics, standardization of the exercise protocol during the vibratory stimulus, type of vibration platform and flexion angle of the knees during squats carried out on the vibration platform.

Verifying the dose-response curve proposal in this study, it can be noted that the protocol of WBV4 (567.91 m.s $\mathrm{s}^{-2}$ ) was not able to promote significant increases in muscle performance tests. We propose that the occurrence of these outcomes (lack of improvement in muscle performance after WBV at very high intensities) could be influenced by a possible framework for muscle fatigue promoted by the squatting exercise. In the literature, there is an inference that the addition of the WBV may predispose individuals to additional exercise loading ${ }^{20,25,34-36}$. It is possible that the maximum acceleration values produced by the protocol of WBV4 (567.91 m.s $\mathrm{s}^{-2}$ ) could overload an excessively muscular system, resulting in a likely situation of fatigue blocking the occurrence of improvement in muscle performance.

Noting that the maximum acceleration values obtained in vibration protocols of WBV2 (159.73 $\left.\mathrm{m}_{\mathrm{s}} \mathrm{s}^{-2}\right)$ and WBV3 (319.45 $\mathrm{m} . \mathrm{s}^{-2}$ ) can promote improvements in a muscle power test, we believed it was worth checking the influence of these interventions on the performance of high intensity and short duration (Wingate test). It appears in our study that the protocol of WBV2 (159.73 m. $\left.\mathrm{s}^{-2}\right)$ was able to promote improvement in the relative power and the protocol of WBV3 $\left(319.45 \mathrm{~m} \cdot \mathrm{s}^{-2}\right)$ increased related work. This result suggests that the WBV can be beneficial for improving the high-intensity cycling anaerobic performance. In agreement with these findings, Avelar et al. ${ }^{1}$ demonstrated that WBV improves the high intensity and short duration cycle performance. This finding indicates that WBV as an ergogenic action in this type of exercise could be suitable primarily for track competition cycling consisting of 200-m and 500-m sprints. Furthermore, Oosthuyse et al. ${ }^{36}$ evaluated the effect of the addition of WBV on aerobic and anaerobic cycle training and observed an improvement of $6 \%$ in peak power and $2 \%$ in mean power during WT from pre- to post-intervention. This study suggested WBV training as an attractive supplement to improve anaerobic power without augmenting the muscle mass of road cyclists.

Despite the benefits obtained by WBV on tests of muscular power and anaerobic power of the lower limbs, the addition of WBV has not been able to increase the handgrip strength in the upper limb. Our findings are in agreement with certain studies in which it was not possible to verify an increase in muscle performance of the upper limbs, even with an increase in performance for the lower limbs ${ }^{8,10}$. Cochrane and Stannard ${ }^{8}$ quantified the acute effect of WBV training on countermovement vertical jump, grip strength, and flexibility performance and found that WBV was able to improve both the vertical jump and flexibility without changes in grip strength. The authors inferred that muscle groups less proportionally exposed to vibration do not exhibit physiological changes that potentiate muscular performance. The magnitude of the vibratory stimulus affects the joints and may reflect subsequent performance on the test, as the more proximal joints receive the majority of vibration acceleration and physiological responses are related to the values of maximum acceleration. Given this situation, it is possible that muscle groups closer to the base of the platform would be 
more responsive during the subsequent muscular responses. Thus, it can be assumed that no increase in upper limb muscle performance was evident because the transmission of vibration was totally damped by the lower extremity.

The present study has limitations, and the results must be interpreted within the context of the experimental design. These findings cannot be generalized to the entire sedentary population, as the volunteers in this study have a frequent and high level of weekly physical activity.

\section{Conclusions}

These results suggest that lower limb muscular performance and anaerobic power are responsive to vibration accelerations WBV2 (159.73 m.s-2) and WBV3 (319.45 m.s-2).

\section{References}

1. Avelar NC, Costa SJ, da Fonseca SF, Tossige-Gomes R, Gripp FJ, Coimbra CC, et al. The effects of passive warm-up vs. Wholebody vibration on high-intensity performance during sprint cycle exercise. J Strength Cond Res. 2012;26(11):2997-3003.

2. Xu L, Rabotti C, Mischi M. Analysis of vibration exercise at varying frequencies by different fatigue estimators. IEEE Trans Neural Syst Rehabil Eng. 2016;24(12):1284-93.

3. Cochrane DJ, Stannard SR, Firth EC, Rittweger J. Acute wholebody vibration elicits post-activation potentiation. Eur J Appl Physiol. 2010;108(2):311.

4. Lamont HS, Cramer JT, Bemben DA, Shehab RL, Anderson MA, Bemben MG. The acute effect of whole-body low-frequency vibration on countermovement vertical jump performance in college-aged men. J Strength Cond Res. 2010;24(12):3433-42.

5. Lovell R, Midgley A, Barrett S, Carter D, Small K. Effects of different half $\square$ time strategies on second half soccer $\square$ specific speed, power, and dynamic strength. Scand J Med Sci Sports. 2013;23(1):105-13.

6. Siu PM, Tam BT, Chow DH, Guo J-Y, Huang Y-P, Zheng Y-P, et al. Immediate effects of 2 different whole-body vibration frequencies on muscle peak torque and stiffness. Arch Phys Med Rehabil. 2010;91(10):1608-15.

7. Maeda N, Urabe Y, Sasadai J, Miyamoto A, Murakami M, Kato J. Effect of Whole-Body-Vibration Training on Trunk-Muscle Strength and Physical Performance in Healthy Adults: Preliminary Results of a Randomized Controlled Trial. J Sport Rehabil. 2016;25(4):357-63.

8. Cochrane DJ, Stannard SR. Acute whole-body vibration training increases vertical jump and flexibility performance in elite female field hockey players. Br J Sports Med. 2005;39(11):860-5.

9. Bullock N, Martin DT, Ross A, Rosemond CD, Jordan MJ, Marino FE. Acute effect of whole-body vibration on sprint and jumping performance in elite skeleton athletes. The J Strength Cond Res. 2008;22(4):1371-4.

10. Dabbs NC, Brown LE, Coburn JW, Lynn SK, Biagini MS, Tran TT. Effect of whole-body vibration warm-up on bat speed in women softball players. J Strength Cond Res. 2010;24(9):2296-9.
11. Kelly SB, Alvar BA, Black LE, Dodd DJ, Carothers KF, Brown LE. The effect of warm-up with whole-body vibration vs. cycle ergometry on isokinetic dynamometry. J Strength Cond Res. 2010;24(11):3140-3.

12. Cardinale M, Bosco C. The use of vibration as an exercise intervention. Exerc Sport Sci Rev. 2003;31(1):3-7.

13. Cochrane D. Vibration exercise: the potential benefits. Int J Sports Med. 2011;32(02):75-99.

14. Prisby RD, Lafage-Proust M-H, Malaval L, Belli A, Vico L. Effects of whole-body vibration on the skeleton and other organ systems in man and animal models: what we know and what we need to know. Ageing Res Rev. 2008;7(4):319-29.

15. Cheng C-F, Hsu W, Lee C, Chung PK. Effects of the different frequencies of whole-body vibration during the recovery phase after exhaustive exercise. J Sports Med Phys Fitness. 2010;50(4):407-15.

16. Rønnestad BR. Acute effects of various whole-body vibration frequencies on 1RM in trained and untrained subjects. J Strength Cond Res. 2009;23(7):2068-72.

17. Ritzmann R, Gollhofer A, Kramer A. The influence of vibration type, frequency, body position, and additional load on the neuromuscular activity during whole-body vibration. Eur J Appl Physiol. 2013;113(1):1-11.

18. Turner AP, Sanderson MF, Attwood LA. The acute effect of different frequencies of whole-body vibration on countermovement jump performance. J Strength Cond Res. 2011;25(6):1592-7.

19. Marín PJ, Bunker D, Rhea MR, Ayllón FN. Neuromuscular activity during whole-body vibration of different amplitudes and footwear conditions: implications for prescription of vibratory stimulation. J Strength Cond Res. 2009;23(8):2311-6.

20. Cochrane DJ, Stannard SR, Sargeant AJ, Rittweger J. The rate of muscle temperature increase during acute whole-body vibration exercise. Eur J Appl Physiol. 2008;103(4):441-8.

21. Clark R, Edholm OG. Man and his thermal environment: Arnold London; 1985.

22. Inbar O, Bar-Or O, Skinner JS. The Wingate anaerobic test: John Wiley \& Sons; 1996.

23. Batista M, Wallerstein L, Dias R, Silva R, Ugrinowitsch C, Tricoli V. Effects of training with vibratórys platforms. R Bras Ci e Mov. 2007;15(3):103-13.

24. Lorenzen C, Maschette W, Koh M, Wilson C. Inconsistent use of terminology in whole-body vibration exercise research. J Sci Med Sport. 2009;12(6):676-8.

25. Avelar NCP, Simão AP, Tossige-Gomes R, Neves CDC, RochaVieira E, Coimbra CC, et al. The effect of adding whole-body vibration to squat training on the functional performance and self-report of disease status in elderly patients with knee osteoarthritis: a randomized, controlled clinical study. J Altern Complement Med. 2011;17(12):1149-55.

26. Sale DG. Post activation potentiation: role in human performance. Exerc Sport Sci Rev. 2002;30(3):138-43.

27. Cormie P, Deane RS, Triplett NT, McBride JM. Acute effects of whole-body vibration on muscle activity, strength, and power. J Strength Cond Res. 2006;20(2):257-61.

28. Sayers SP, Harackiewicz DV, Harman EA, Frykman PN, Rosenstein MT. Cross-validation of three jump power equations. Med Sci Sports Exerc. 1999;31(4):572-7. 
29. Caporrino F, Faloppa F, Santos J, Réssio C, Soares F, Nakashima L. Populational study of the grip force with $\operatorname{Jamar}{ }^{\circledR}$ dynamometer. Rev Bras Ortop. 1998;33:150-4.

30. Cohen J. Statistical Power Analysis for the Behavioral Sciences. 2nd ed. Hillsdale (NJ): Lawrence Erlbaum Associates; 1988. $567 \mathrm{p}$.

31. Kvorning T, Bagger M, Caserotti P, Madsen K. Effects of vibration and resistance training on neuromuscular and hormonal measures. Eur J Appl Physiol. 2006;96(5):615-25.

32. Ross A, Leveritt M. Long-term metabolic and skeletal muscle adaptations to short-sprint training. Sports Medicine. 2001;31(15):1063-82.

33. Burnley M, Doust JH, Jones AM. Effects of the prior warm-up regime on severe-intensity cycling performance. Med Sci Sports Exerc. 2005;37(5):838-45.

34. Rittweger J, Mutschelknauss M, Felsenberg D. Acute changes in neuromuscular excitability after exhaustive whole-body vibration exercise as compared to exhaustion by squatting exercise. Clin Physiol Funct Imaging. 2003;23(2):81-6.

35. Roelants M, Verschueren SM, Delecluse C, Levin O, Stijnen $\mathrm{V}$. Whole-body-vibration-induced increase in leg muscle activity during different squat exercises. J Strength Cond Res. 2006;20(1):124-9.

36. Oosthuyse T, Viedge A, McVeigh J, Avidon I. Anaerobic power in road cyclists is improved after 10 weeks of whole-body vibration training. J Strength Cond Res. 2013;27(2):485-94.

\section{Acknowledgments}

SBFis, FAPEMIG, CNPq and CAPES.

\section{Corresponding author}

Nubia Carelli Pereira de Avelar

Federal University of Santa Catarina, Department of Health Sciences (DCS). Rodovia Governador Jorge Lacerda, 3201 - Km 35,4, Jardim das Avenidas, 88906-072. Araranguá/SC. Brazil. Telephone: +55 (48) 37212167.

Email: nubia.carelli@ufsc.br

Editor: Angelina Zanesco. UNESP/Rio Claro, SP, Brazil

Manuscript received on October 18, 2019

Manuscript accepted on July 27, 2020

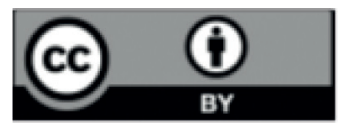

Motriz. The Journal of Physical Education. UNESP. Rio Claro, SP, Brazil - eISSN: 1980-6574 - under a license Creative Commons - Version 4.0 